DOI 10.1007/s00417-004-0996-6

\author{
André A. Dosso \\ Elisabeth Rungger-Brändle
}

\section{Bilateral corneal perforation in familial amyloidotic polyneuropathy}

Received: 8 April 2004

Revised: 23 June 2004

Accepted: 3 July 2004

Published online: 28 September 2004

(C) Springer-Verlag 2004

\author{
A. A. Dosso - E. Rungger-Brändle \\ Cell Biology Laboratory, \\ University Eye Clinic, \\ Geneva, Switzerland
}

\section{A. A. Dosso ( $)$}

Clinique d'Ophtalmologie,

22, rue Alcide Jentzer, 1211 Genève 14 Switzerland

e-mail: andre.dosso@hcuge.ch

Tel.: +41-22-3828363

Fax: +41-22-3828382

\begin{abstract}
Purpose: We report the progression of bilateral central perforating ulceration in the cornea of a patient with familial amyloidotic polyneuropathy (FAP), also known as hereditary Portuguese amyloidosis, who received two corneal grafts in an interval of 6 years. The pathology of the original host and the grafted cornea is described. Methods: Overall histology and immunolocalization of transthyretin, amyloid beta $(\mathrm{A} \beta)$, and epithelial and inflammatory markers were performed. Results: Corneal sensitivity and tear film were reduced. The grafted but not the original tissue contained amyloid deposits with transthyretin immunoreactivity. Epithelial and stromal thinning was accentuated in the graft, with epithelial dysplasia, hyperproliferation, and parakeratosis. Abundance of basement membrane material in hyper-
\end{abstract}

proliferative regions suggested recurrent attempts of wound healing. Activated keratocytes, ingrowth of vessels, infiltrated inflammatory, and immune cells reflect both acute and chronic inflammation. Conclusion: Amyloid deposits may progressively reduce corneal sensitivity and damage epithelium and stroma. Corneal neuropathy, together with impaired tear film, may entail the pathology of dry eyes as a bystander effect, contributing to exacerbation of epithelial injury, deregulated proliferation, and parakeratosis. Once established, both acute and chronic inflammation may sustain progression of the corneal pathology.

Keywords Familial amyloidotic polyneuropathy - Transthyretin . Amyloid-beta $\cdot$ Cornea $\cdot$ Ulcer

\section{Introduction}

Familial amyloidotic polyneuropathy (FAP) of the Portuguese type is an autosomal dominant disease caused by mutations in the transthyretin (TTR) gene. It is characterized by extracellular amyloid deposits in peripheral nerves and various organs, including the eye [5]. The peripheral sensory neuropathy consists of paraesthesia and loss of thermal and pain sensation in the extremities [3] and in the cornea (this study).

Among the clinical manifestations in the eye of FAP patients, vitreous opacities are frequent $[8,9]$. In tissues surrounding the anterior chamber, perivascular deposits are considered to obstruct aqueous outflow, eventually causing secondary glaucoma $[7,10]$. Corneal perforation was reported in two cases $[1,4]$. In this study, we present the histopathology of a case of FAP with bilateral, recurrent corneal perforation.

\section{Case report}

In 1991, a 31-year-old Portuguese man presented a peripheral autonomic neuropathy and gastrointestinal dysfunction. Analysis by RT-PCR confirmed mutation in the TTR gene (Val30Met). In 1994, the patient complained about bilateral visual impairment. Vision was reduced to digital numeration, and bilateral, central, perforating corneal ulceration was diagnosed. Corneal grafts were performed on both eyes. The patient was treated with oral cyclosporine
} 

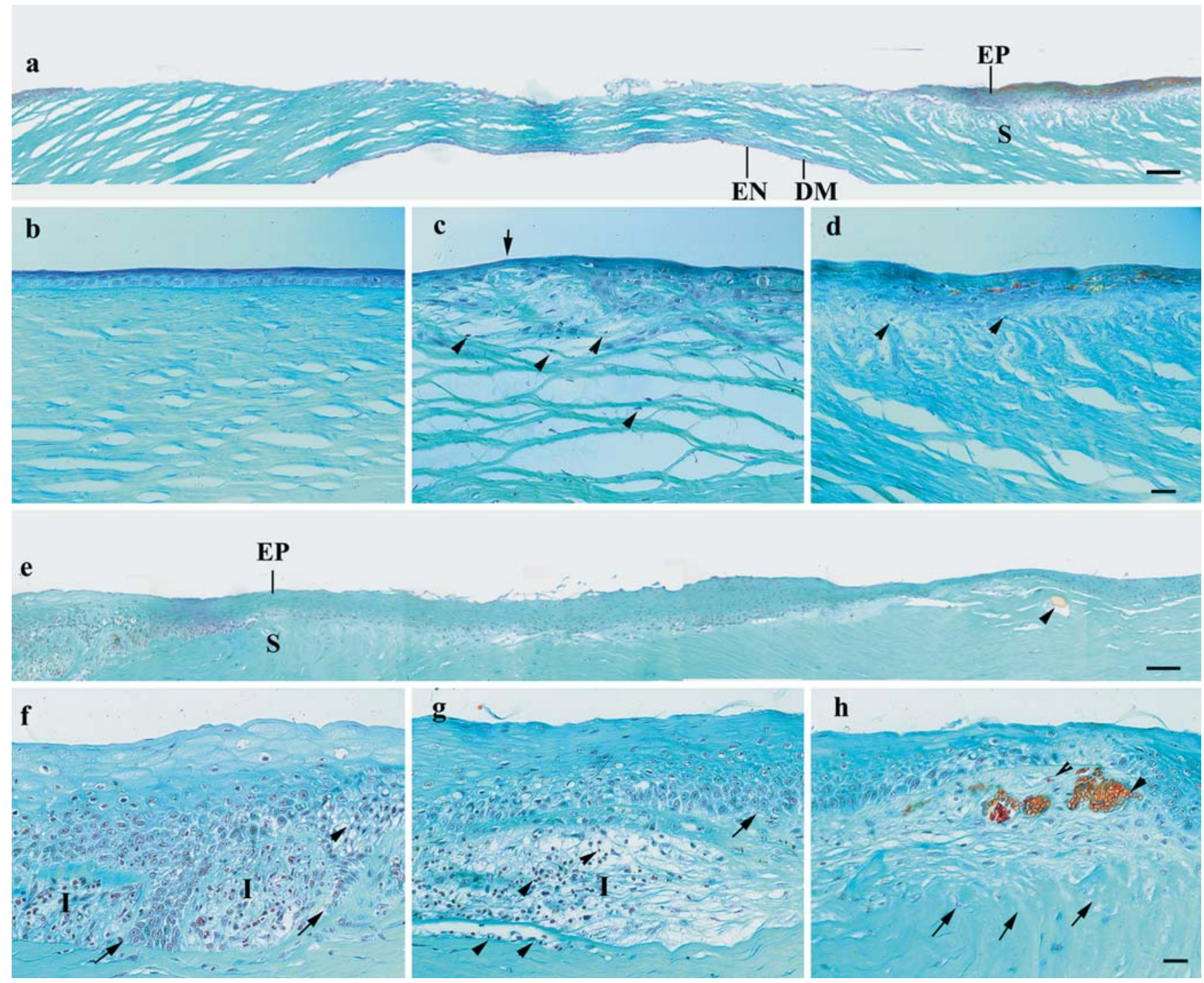

Fig. 1 Changes in the original (a-d) and grafted (e-h) corneal button, replaced after 6 years. Trichrom Masson-Goldner staining. a Absence of epithelium (EP) and thinning of stroma (S) in central cornea. Descemet's membrane (DM) and endothelium (EN) are intact. Bar, $20 \mu \mathrm{m}$. b Periphery with normal epithelium, stroma, and Bowman's layer. c,d Subepithelial stroma with infiltrated inflammatory cells (arrowheads) and locally thinned epithelium (c, arrow), where Bowman's layer is missing. Bar, $10 \mu \mathrm{m}$. e Region close to a perforated area with suture stitch (arrowhead). Bar,

(250 mg daily) and topical antibiotics (chloramphenicol), steroids, nonpreserved lubrificants, and vitamin A. During the following years, vision remained stable at $10 / 20$ in both eyes. No clinical signs of graft rejection were observed. The compliance of the patient was not optimal, and a cyclosporinemia in the therapeutic range was not obtained. Severe hypoesthesia and keratoconjunctivitis sicca were noted. In 2000, the patient presented a recurrent corneal perforation on the right eye with the characteristics of a neurotrophic ulcer. A second transplantation was performed.
$20 \mu \mathrm{m}$. f-h Higher magnifications of $e$. Bar, $10 \mu \mathrm{m}$. f Stacks of hyperproliferative (arrows) and dysplastic (arrowhead) epithelial cells between inflammatory foci (I) in subepithelium. Large nuclei in surface layers indicate parakeratosis. $\mathbf{g}$ Disintegration of upper stroma in inflamed regions (arrowheads). d,h Neovessels and extravasated erythrocytes (arrowheads) in the subepithelial granulation tissue. Note perpendicular orientation of stromal layers (arrows).

\section{Methods}

Overall histology was performed with trichrome Masson-Goldner and hematoxylin-eosin staining. Amyloid deposits were detected with Congo Red and thioflavine. TTR was identified with a rabbit polyclonal antibody (1:1,000; DAKO, Zug, Switzerland) and revealed by biotin-streptavidin peroxidase. The following antibodies were used on frozen sections: mouse monoclonal anti-pan-cytokeratin (1:5; clone LU5, Roche, Rotkreuz, Switzerland), mouse monoclonal anticytokeratin 14 (1:10; clone LL002, gift of Dr B. Lane, Dundee, UK), goat polyclonal anti-A $\beta$ protein (1:25; Santa 


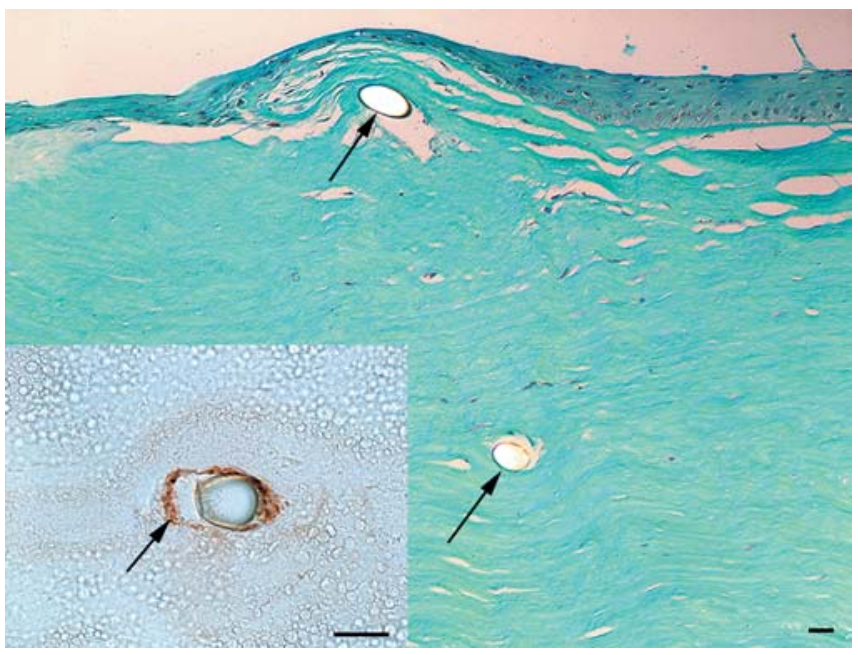

Fig. 2 Sutural stitches (arrows) in the stroma of the graft surrounded by a rim of immunoreactive transthyretin (TTR) (arrow in insert). Bar, $10 \mu \mathrm{m}$.

Cruz, LabForce, Nunningen, Switzerland), mouse monoclonal antivimentin (1:100; clone 3B4, Progen Biotechnik, Heidelberg, Germany), mouse monoclonal anti- $\alpha$ smooth-muscle actin $(1: 10$; gift of Dr. G. Gabbiani, Geneva, Switzerland), mouse monoclonal anti-CD44 (1:50; Pharmingen, BD Biosciences, Basel, Switzerland), mouse monoclonal anti-CD45 (1:50, Santa Cruz, LabForce), and rabbit polyclonal antilaminin (1:50; Sigma, Buchs, Switzerland). Secondary antibodies were coupled with Texas Red (1:200, Jackson Immunoresearch, Milan Analytica, La Roche, Switzerland). Sections were viewed with a confocal laser scanning microscope (Zeiss LSM 410), and images were treated with Imaris software (Bitplane, Zürich, Switzerland). Normal cornea was from a 16-year-old female multidonor.

\section{Results}

Light microscopy of the original corneal button revealed thinning of stroma and lack of epithelium in the centralmost cornea (Fig. 1a). The periphery appeared normal (Fig. 1b). Locally, the subepithelial stroma was replaced by granulation tissue (Fig. 1c) and oriented perpendicularly to the corneal surface (Fig. 1d). Bowman's layer was missing, but Descemet's membrane and endothelium appeared normal throughout. The removed first graft presented severe exacerbation (Fig. 1e). Epithelial thickness was irregular, and in ulcerated regions, epithelium and stroma were lacking, leaving Descemet's membrane and endothelium bare. Subepithelial granulation tissue was intersected by hyperproliferating epithelial stacks (Fig. 1f). Cell clusters appeared dysplastic and, in surface layers, parakeratotic with persistence of large nuclei (Fig. 1f). Occasionally, several basement membranes were noted (Fig. 1f-h) that heavily expressed laminin (not shown). Inflammatory infiltrates with neovessels and extravasated erythrocytes (Fig. 1h) were woven into arrays of extracellular matrix, reflecting destruction of the subepithelial stroma.

Stromal amyloid deposits surrounded sutural stitches (Figs. 1e, 2a). By Congo Red staining viewed under polarized light, they produced a rim of red-green dichroism (not shown). Reaction with thioflavin disclosed a similar rim (not shown) that was immunoreactive for TTR (Fig. 2b).

Staining of the graft for pan-cytokeratin revealed irregular thickness of the epithelium (Fig. 3). In regions of large thickness, nuclei persisted in cells of the surface layers, characteristic of parakeratosis. In perforated regions, staining was poor in cells apposing directly onto Descemet's membrane.

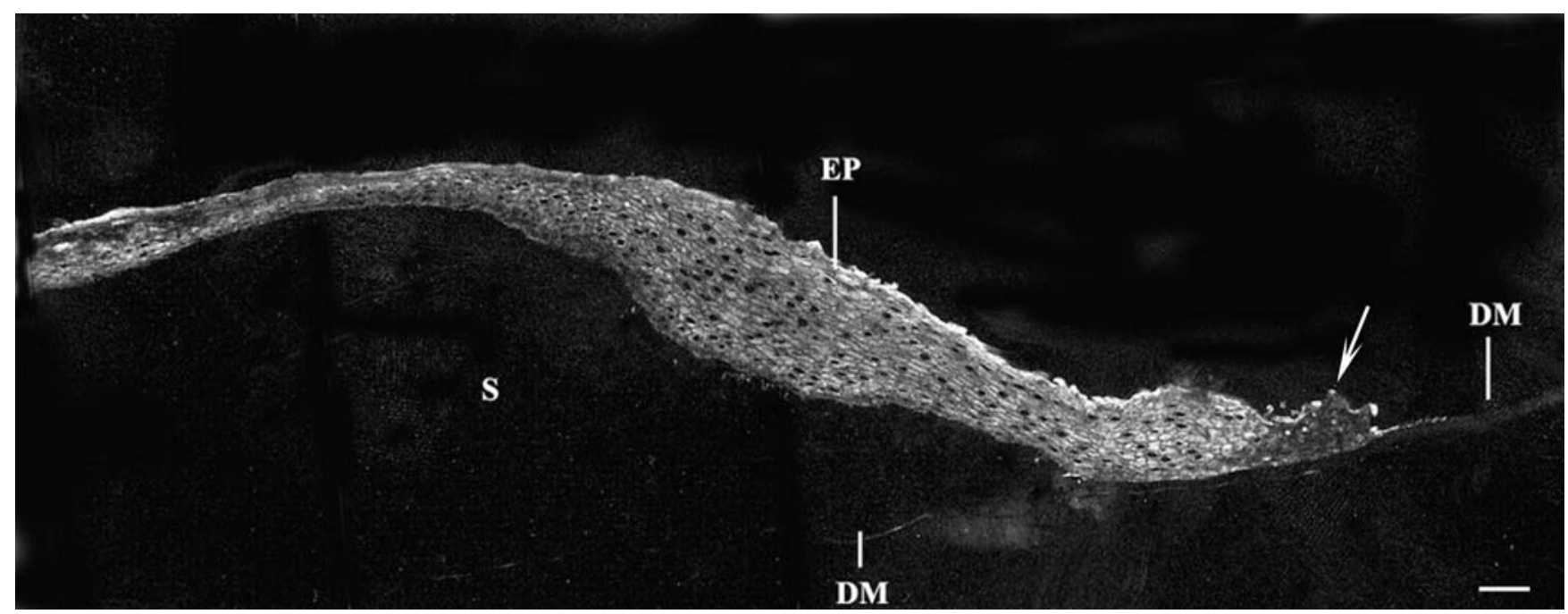

Fig. 3 Unequal thickness of the epithelium (EP) in the graft, revealed by immunofluorescence staining for pan-cytokeratin. The protrusion growing on Descemet's membrane (DM) in the ulcerated center (right) stains weakly. $S$, stroma; bar, $100 \mu \mathrm{m}$. 

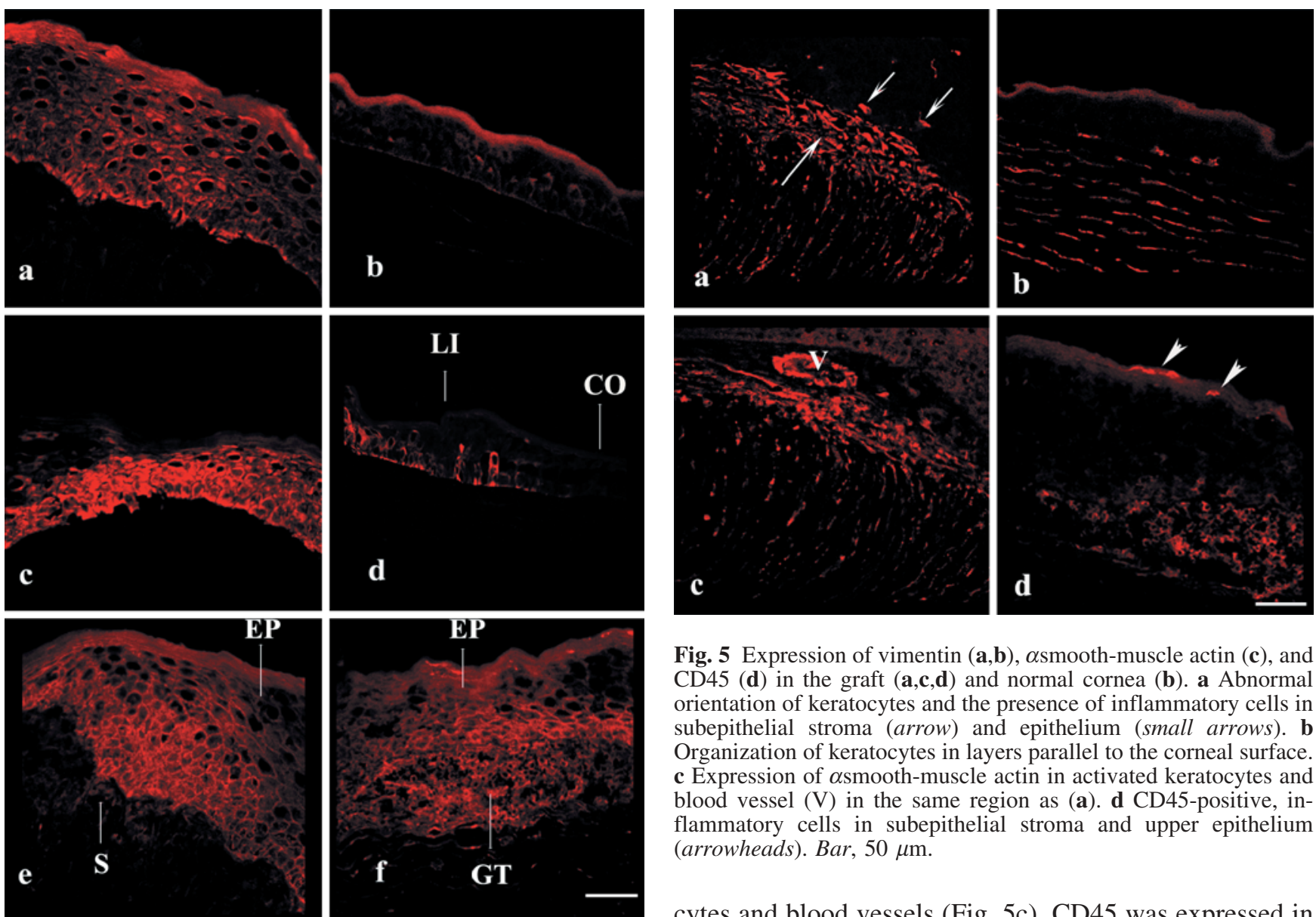

Fig. 4 Immunolocalization of cytokeratin $14(\mathbf{a}, \mathbf{b}), \mathrm{A} \beta$ (c,d), and CD44 (e,f) in the graft $(\mathbf{a}, \mathbf{c}, \mathbf{e}, \mathbf{f})$, in normal central cornea (b), and corneolimbal transition zone (d). a,b Cytokeratin 14 is expressed throughout the epithelium of the graft (a) and in basal cells of normal cornea (b). c,d A $\beta$ is expressed in basal and several suprabasal layers of the graft (c) and in basal cells of normal limbus (f, LI) but not in normal cornea (CO). e,f CD44 is expressed in cytoplasm and plasma membranes throughout the epithelium (EP), in subepithelial stroma (e, S), and in cells of granulation tissue (GT) and upper epithelial layers (f). Bar, $50 \mu \mathrm{m}$.

Cytokeratin 14, a marker of basal epithelial cells in normal cornea (Fig. 4b), was expressed throughout all layers of the graft (Fig. 4a). Staining for $\mathrm{A} \beta$ protein was enhanced in basal and suprabasal cells (Fig. 4c). This is unusual, as $\mathrm{A} \beta$ is normally expressed in the limbus but not in the cornea (Fig. 4d). Staining for CD44, transiently expressed during reepithelialization, was noted throughout epithelium and stroma (Fig. 4e), in granulation tissue, as well as inflammatory cells in the uppermost epithelial layers (Fig. 4f).

In regions with granulation tissue, antibodies to vimentin revealed keratocytes perpendicularly oriented to the corneal surface (Fig. 5a) whereas, in normal cornea, they orient in parallel layers (Fig. 5b). Staining for $\alpha$ smooth-muscle actin was prominent in activated kerato-

Fig. 5 Expression of vimentin (a,b), $\alpha$ smooth-muscle actin (c), and CD45 (d) in the graft (a,c,d) and normal cornea (b). a Abnormal orientation of keratocytes and the presence of inflammatory cells in subepithelial stroma (arrow) and epithelium (small arrows). b Organization of keratocytes in layers parallel to the corneal surface. c Expression of $\alpha$ smooth-muscle actin in activated keratocytes and blood vessel (V) in the same region as (a). d CD45-positive, inflammatory cells in subepithelial stroma and upper epithelium (arrowheads). Bar, $50 \mu \mathrm{m}$.

cytes and blood vessels (Fig. 5c). CD45 was expressed in inflammatory cells in granulation tissue and uppermost epithelial layers (Fig. 5d). By classical hematoxylin-eosin staining (not shown), most of the cells forming the granulation tissue were lymphocytes and macrophages interspersed with numerous polymorphonuclear leucocytes (PMNs).

\section{Discussion}

The histological comparison between host cornea and graft revealed that transplantation did not prevent progression of disease. This must be due to progressive replacement of the genetically normal graft by cells from the mutant host limbus and by ingrowth of vessels during the inflammatory process. In the original host button, erosion was confined to the central-most cornea whereas in the graft, extended areas were damaged: tissue destruction was worse, and stromal amyloid deposits containing TTR, not noticed in the original button, had formed around sutural stitches, suggesting that a cytotoxic effect by amyloid may clinically be recognized early on as thinning of epithelium and stroma.

Despite (poorly complied) anti-inflammatory medication, massive granulation tissue had formed in the sub- 
epithelium of the graft. CD44-positive and CD45-positive cells, neovessels, and activated keratocytes, recognized by the expression of $\alpha$ smooth-muscle actin, were noted. Strikingly, the simultaneous presence of PMNs and mononuclear-cell infiltrates containing numerous lymphocytes indicate that concomitant acute and chronic inflammation had occurred. Immunoreactivity for CD44 and CD45 was also noted in the outermost epithelium, suggesting that inflammatory and possibly also immune cells from the tear film were retained. It is thus likely that, besides the early cytotoxic effect caused by amyloid deposits, inflammation was maintained by additional insults, such as decreased corneal sensitivity and/or impaired tear secretion evoked by deregulation of the lacrimal glands. In the present case, both features were noticed, accounting for the pathology of dry eyes [2]. Ongoing mechanical damage of the corneal surface may be a major cause for continuous recruitment and retention of inflammatory cells, enhancing both local deregulation of epithelial growth and destruction of epithelium and stroma. Besides acute and chronic inflammation, recurrent but abortive attempts of epithelial wound healing [6] were made, as documented by the presence of a multilayered basement membrane, epithelial hyperplasia, and parakeratosis with persistent expression of $\mathrm{A} \beta$ protein, cytokeratin 14 , and
CD44, monitored here because it is transiently expressed during reepithelialization [11]. Our observation marks parakeratosis as an important condition for fragility and insufficient protection of the epithelium, rendering the cornea vulnerable to infection.

In conclusion, we propose the following scenario for the pathogenesis of the FAP cornea: Initially, formation of amyloid deposits creates a cytotoxic milieu that damages sensory nerves, epithelium, and stroma. Corneal neuropathy and an impaired tear film may account for damage of the epithelial surface and disturbances in hydration and ionic homeostasis. Both persistent mechanical damage and cytotoxicity of monocytic infiltrates cause continuous recruitment of PMNs and set up the conditions for excessive tissue destruction and recurrent attempts of wound healing. Overexpression of bystander molecules such as $\mathrm{A} \beta$ protein, $\mathrm{CD} 44$, and inflammatory cytokines may force the epithelium into hyperproliferation and parakeratosis, further weakening protection of the corneal surface.

Acknowledgements We thank M. Alain Conti for his skillful technical assistance and Dr. G.-P. Pizzolato for helping us in identifying amyloid deposits. This study was supported by the Swiss National Foundation (grant 3100-055106.98) and by the Foundation Provisu.

\section{References}

Abenhaim A, Hoang-Xuan T, GoichotBonnat L, Dhermy P, Pouliquen Y (1988) A case of spontaneous corneal perforation in De Andrade's portuguese amyloidosis. Bull Soc Ophtalmol Fr 88:223-226

Baudouin C (2001) The pathology of dry eye. Surv Ophthalmol 45(Suppl 2):S211-S220

Coutinho P, Martins da Silva A, Lopes Lima J, Resende Barbosa A (1980) Forty years of experience with type I amyloid neuropathy. Review of 483 cases. In: Glenner GG, Costa PP, Freitas AF (eds) Amyloid and amyloidosis. Excerpta Medica, Amsterdam, p 88

Frossard JL, Donati G, Reymond JM (1996) Portuguese amyloidosis: a case of spontaneous bilateral corneal perforation. Am J Med 101:562
Haraoka K, Ando Y, Ando E, Sandgren O, Hirata A, Nakamura M, Terazaki H, Tajiri T, Tanoue Y, Sun X, Okabe H, Tanihara H (2002) Amyloid deposition in ocular tissues of patients with familial amyloidotic polyneuropathy (FAP). Amyloid 9:183-189

Kim W-J, Helena, MC, Moban RR, Wilson SE (1999) Changes in corneal morphology associated with chronic epithelial injury. Invest Ophthalmol Vis Sci 40:35-42

Kimura A, Ando E, Fukushima M, Koga T, Hirata A, Arimura K, Ando Y, Negi A, Tanihara H (2003) Secondary glaucoma in patients with familial amyloidotic polyneuropathy. Arch Ophthalmol 121:351-356
Koga T, Ando E, Hirata A, Fukushima M, Kimura A, Ando Y, Negi A, Tanihara $\mathrm{H}$ (2003) Vitreous opacities and outcome of vitreous surgery in patients with familial amyloidotic polyneuropathy. Am J Ophthalmol 135:188-193

Munar-Ques M, Salva-Ladaria L, MuletPerera P, Sole M, Lopez-Andreu FR, Saraiva MJ (2000) Vitreous amyloidosis after liver transplantation in patients with familial amyloid polyneuropathy: ocular synthesis of mutant transthyretin. Amyloid 7:266-269

Nelson GA, Edward DP, Wilensky JT (1999) Ocular amyloidosis and secondary glaucoma. Ophthalmology 106:1363-1366

Yu FX, Guo J, Zhang Q (1998) Expresssion and distribution of adhesion molecule CD44 in healing corneal epithelia. Invest Ophthalmol Vis Sci 39:710-717 